Dr. med. Harald Haas, Bern, Schweiz

\section{Anthroposophische Psychotherapie und Psychologie}

In den drei Beiträgen zum Schwerpunktthema «Anthroposophische Psychotherapie und Psychologie» sollen Aspekte angesprochen werden, die eine grössere klinische Bedeutung haben [1-3]. Dabei besteht die Voraussetzung, dass zum Verständnis des Folgenden ein anderes Menschenbild als das heute übliche in der Psychiatrie und Psychotherapie/Psychologie als Grundlage genommen wird. Auch wenn die Schweizerische Psychiatriegesellschaft (SGPP) und die Schweizerische Gesellschaft für Psychosomatik (SAPPM) das bio-psycho-soziale Konzept nach G.L. Engel [4] zur Grundlage hat, so weist es doch den Mangel auf, dass die verschiedenen Dimensionen des Menschseins hiermit nicht stimmig miteinander verbunden werden können. Darauf hat der Philosoph Peter Bieri, der als Autor unter dem Namen Pascal Mercier bekannt wurde, in den nach ihm benannten Bieri-Trilemma bezüglich des sogenannten Leib-Seele-Problems hingewiesen [5].

Dabei gibt es schon seit der klassisch-romantischen Philosophie eine Tradition, welche die vier Ebenen von Körper, Leib, Seele und Geist beschreibt. Ein hervorragender Vertreter dieser Anschauung ist der Schweizer Philosoph und Arzt Ignaz Paul Vital Troxler (1780-1866), dessen Jubiläum zum 100. Todestag im letzten Jahr begangen wurde. 1812, noch bevor er 1834 erster Philosophieprofessor der neu gegründeten Berner Universität wurde, hat er in seinem Werk «Blicke in das Wesen des Menschen» [6] die Viergliederung des Menschen dargestellt. Später in der
Universität Bern begründete er in den «Vorlesungen über Philosophie» [7] eine Erkenntnistheorie des Geistes, die er Anthroposophie nannte. Anschliessend an dieses Menschenbild entwickelte Rudolf Steiner seine «anthroposophische Geisteswissenschaft», die nunmehr seit 100 Jahren mit dem Beginn der Mistelbehandlung durch Ita Wegman [8] in die Medizin Eingang gefunden hat.

Die heutige Praxis der anthroposophischen Psychotherapie und Psychologie kann sich sehr gut mit dem Konzept der Salutogenese (siehe [1]) - nach dem israelisch-amerikanischen Soziologen Aaron Antonovsky - verbinden und liegt allen Artikeln dieses Schwerpunktthemas zu Grunde. Diese medizinisch-psychologische Betrachtungsweise hat sich vor allem in Mitteleuropa und weniger in ihrem Ursprungsland USA bzw. in der englischsprachigen Literatur weiterentwickelt. Die im ersten Artikel [1] beschriebene Gruppentherapie der Nervosität (im Anschluss an Rudolf Steiners Vortrag «Nervosität und Ichheit» vom 11.1.1912 in München [9]), die auch im Sinne der Formulierungen Rudolf Steiners aus dem Salutogenese-Konzept entwickelt wurde, hat enge Beziehungen zu den heutigen Achtsamkeitstherapien [10], da beide Therapieansätze in der Tradition der buddhistischen Praxis stehen. Dabei stammt der Begriff der Nervosität vom amerikanischen Neurologen George Miller Beard (1839-1883), der die zuvor von ihm benannte «Neurasthenie» als «American Nervousness» bezeichnete $[11,12]$. Dass die Meditation in der Anthroposophischen Medizin eine vielfältige Anwendung findet, zeigt ein Praxisbuch für Ärzte, Therapeuten, Pflegende und Patienten, das von Michaela Glöckler herausgegeben wurde [13].

Der Artikel von Martina Haeck [2] über die stationäre Behandlung von Schlafstörungen zeigt eine weitere praktische Relevanz der anthroposophischen Anschauungen auf, die sich sehr gut mit dem Konzept der Salutogenese verbinden lässt. Hierbei wird vor allem durch das Mittel der Psychoedukation eine für den Patienten nachvollziehbare Anwendung der geisteswissenschaftlich-menschenkundlichen Zusammenhänge bezüglich seines Leidens möglich. Dabei lassen sich die naturwissenschaftlichen Resultate sehr gut mit diesem Therapieverständnis verbinden.

Vertiefend zum Thema Schlafmedizin kann noch auf ein Themenschwerpunkt-Heft der anthroposophisch-medizinischen Zeitschrift Der Merkurstab unter dem Titel «Schlaf und Schlaflosigkeit» hingewiesen werden [14].

Die Artikelserie wird abgerundet mit einem Beitrag von Christian Schopper [3] zur anthroposophisch orientierten Psychoonkologie. Auch hier zeigt sich, wie er betont, dass

\section{KARGER}

(C) 2017 S. Karger GmbH, Freiburg

Fax +497614520714
Dr. med. Harald Haas

Psychiatrie und Psychotherapie FMH

Anthroposophische Medizin VAOAS und GAÄD

Anthroposophische Psychotherapie DtGAP

Münstergasse 68, 3011 Bern, Schweiz

harald.haasbe@bluewin.ch 
es sich bei den anthroposophischen Therapiekonzepten nicht um eine komplementäre Behandlung, sondern um eine integrative Medizin handelt. Dabei weist er darauf hin, dass die verschiedenen psychoonkologisch tätigen Autoren sehr gut mit dem anthroposophischen Menschenbild und der Idee der Salutogenese verbunden werden können. In der Onkologie und Palliativmedizin ist es besonders bedeutend, dass die integrative Praxis in die medizinisch-psychologische Praxis Einzug hält. Gerade für die Palliativmedizin ist mit der anthroposophischen Psychoonkologie eine we- sentliche Erweiterung durch das Einbeziehen der «nachtodlichen Situation» für den Umgang mit den Patienten möglich.

Zuletzt bleibt noch darauf hinzuweisen, dass es durch die Professur für anthroposophisch erweiterte Medizin an der Universität Bern von Frau Prof. Ursula Wolf möglich ist, Grundlagenforschung sowie praktische Forschung zur anthroposophischen Onkologie durchzuführen [15], was sich unter anderem in einem «Bestcase Portal Onkologie» zeigt.

\section{Literatur}

1 Haas H: Salutogenese und achtsamkeitsorientierte anthroposophische Psychotherapie am Beispiel ambulanter Gruppentherapien bei «Nervosität». Schweiz Z Ganzheitsmed 2017;29: DOI: $10.1159 / 000484462$.

2 Haeck M: Behandlung von Schlafstörungen in der anthroposophischen Klinik. Schweiz Z Ganzheitsmed 2017;29:DOI: 10.1159/000484389.

3 Schopper C: Anthroposophische Psychoonkologie. Schweiz Z Ganzheitsmed 2017;29:DOI: 10.1159/000484451.

4 Engel GL: Psychisches Verhalten in Gesundheit und Krankheit. Bern, Huber, 1976.

5 Bieri P: Analytische Philosophie des Geistes. Königstein, Hain, 1981.

6 Troxler IPV: Blicke in das Wesen des Menschen. Aarau, Sauerländer, 1812.
7 Troxler IPV: Vorlesungen über Philosophie. Über Inhalt, Bildungsgang, Zweck und Anwendung derselben auf's Leben, als Encyclopädie und Methodologie der philosophischen Wissenschaften. Fischer, Bern, 1835.

8 Selg P: Mensch und Mistel - Die Begründung der onkologischen Viscum-Behandlung durch Rudolf Steiner und Ita Wegman. Band 1: 1917-1925. Arlesheim, Verlag des Ita Wegman Instituts, 2016.

9 Steiner R: Nervosität und Ichheit - Stressbewältigung von Innen, Vortrag vom 11.1.1912, eingeleitet von Frank Meyer, Sonderdruck aus Bibliographie-Nr. 143. Dornach, Rudolf Steiner, 1994.

10 Michalak J, Heidenreich T, Williams JMG: Achtsamkeit, ed 1. Fortschritte der Psychotherapie, Band 48. Göttingen, Hogrefe, 2012.
11 Beard GM: A Practical Treatise on Nervous Exhaustion (Neurasthenia). New York, W. Wood \& Co., 1880.

12 Beard GM: American Nervousness, Its Causes and Consequences. New York, G.P. Putnam's Sons, 1880.

13 Glöckler M (Hrsg): Meditation in der Anthroposophischen Medizin - Ein Praxisbuch für Ärzte, Therapeuten, Pflegende und Patienten, ed 1. Berlin, Salumed, 2016.

14 Der Merkurstab: Themenschwerpunkt Schlaf und Schlaflosigkeit. Der Merkurstab 1/2017.

15 Wolf U: Jahresbericht 2016. Professur für anthroposophische erweiterte Medizin an der Universität Bern. www.ikom.unibe.ch/unibe/ portal/fak_medizin/dept_lehremed/inst_kom/ content/e54415/e54416/e54417/e368428/Jahresbericht2016_IKOM-AEM_v3_ger.pdf. 